\section{Associational Fibers}

Melissa J. McGinn

Anatomy \& Neurobiology, Virginia

Commonwealth University School of Medicine,

Richmond, VA, USA

\section{Synonyms}

Arcuate fibers

\section{Definition}

Associational fibers are bundles of white matter that connect various cortical regions within the same cerebral hemisphere. The most prevalent type of white matter tract found in the cortex, associational fibers permit bidirectional communication between different cortical areas, thus allowing the cortex to function as a coordinated whole. Associational fibers predominantly arise from cortical layer III pyramidal neurons and can be classified as either short associational fibers, which connect adjacent gyri within the same lobe, or long associational fibers, interconnecting more distant regions located in different lobes. The major long associational fibers tracts in the brain include the superior longitudinal fasciculus, arcuate fasciculus, uncinate fasciculus, and cingulum.

\section{Cross-References}

- Arcuate Fasciculus

- Association Pathways

- Cerebral Cortex

- Cingulum

- Superior Longitudinal Fasciculus

- White Matter 